\title{
The NuMoon experiment: Lunar detection of cosmic rays and neutrinos with LOFAR.
}

G. K. Krampah, ${ }^{1, *}$ S. Buitink, ${ }^{1,2}$ A. Corstanje, ${ }^{1,2}$ H. Falcke, ${ }^{2,3,4}$ B. M. Hare, ${ }^{5}$ J. R. Hörandel, ${ }^{1,2,3}$ T. Huege,${ }^{1,6}$ K. Mulrey, ${ }^{1}$ P. Mitra, ${ }^{1}$ A. Nelles, ${ }^{7,8}$ H. Pandya, ${ }^{1}$ J. P. Rachen, ${ }^{1}$ O. Scholten, ${ }^{9}$ S. ter Veen, ${ }^{2,4}$ S. Thoudam, ${ }^{10}$ T. N. G. Trinh ${ }^{11}$ and T. Winchen ${ }^{12}$

${ }^{1}$ Astrophysical Institute, Vrije Universiteit Brussel, Pleinlaan 2, 1050 Brussels, Belgium

${ }^{2}$ Department of Astrophysics/IMAPP, Radboud University, P.O. Box 9010, 6500 GL Nijmegen, The Netherlands

${ }^{3}$ Nikhef, Science Park 105, 1098 XG Amsterdam, The Netherlands

${ }^{4}$ Netherlands Institute of Radio Astronomy (ASTRON), Postbus 2, 7990 AA Dwingeloo, The Netherlands

${ }^{5}$ University of Groningen, Kapteyn Astronomical Institute, Groningen, 9747 AD, Netherlands

${ }^{6}$ Institut für Kernphysik, Karlsruhe Institute of Technology (KIT), P.O. Box 3640, 76021, Karlsruhe, Germany

${ }^{7}$ DESY, Platanenallee 6, 15738 Zeuthen, Germany

${ }^{8}$ ECAP, Friedrich-Alexander-University Erlangen-Nürnberg, 91058 Erlangen, Germany

${ }^{9}$ Interuniversity Institute for High-Energy, Vrije Universiteit Brussel, Pleinlaan 2, 1050 Brussels, Belgium

${ }^{10}$ Department of Physics, Khalifa University, PO Box 127788, Abu Dhabi, United Arab Emirates

${ }^{11}$ Department of Physics, School of Education, Can Tho University Campus II, 3/2 Street, Ninh Kieu District, Can Tho City, Vietnam

${ }^{12}$ Max-Planck-Institut für Radioastronomie, Auf dem Hügel 69, 53121 Bonn

E-mail: gkrampah@vub.be

The low flux of ultra-high-energy cosmic rays (UHECRs) makes it challenging to understand their origin and nature. A very large effective aperture is provided by the Lunar Askaryan technique. Particle cascades in a dielectric medium produce radio emission through the Askaryan effect. Ground based radio telescopes are used to search for nanosecond radio pulses that are produced when cosmic rays or neutrinos interact with the Moon's surface. The LOw Frequency ARray (LOFAR) is currently the largest radio array operating at frequencies between $110-190 \mathrm{MHz}$; the optimum frequency range for Lunar signal search and $30-80 \mathrm{MHz}$ for radio detection of air showers. One minute of observation has been carried out with six LOFAR stations beam-formed towards the Moon. In this contribution, we present some preliminary results of the analysis of the data and a complete description of the analysis steps.

$37^{\text {th }}$ International Cosmic Ray Conference (ICRC 2021)

July 12th-23rd, 2021

Online - Berlin, Germany

${ }^{*}$ Presenter 


\section{Introduction}

Ultra-high energy cosmic rays (UHECR) are known to be of mostly extragalactic origin and reach up to energies of $100 \mathrm{EeV}$. At still higher energies, they are expected to be suppressed by interactions with the cosmic microwave background, known as the Greisen-Zatsepin-Kuzmin (GZK) effect [1]. However, in the same energy range, all known potential cosmic accelerators for UHECR are expected to cut off as well [2]. Discerning between the two effects and thus learning about the origin of UHECR would greatly benefit from high-statistics data on cosmic rays above $100 \mathrm{EeV}$ - a goal which is difficult to reach with Earth-bound experiments because of the extremely low flux of these particles.

A way out could be the Lunar Askaryan effect, which may allow to observe cosmic rays above a sensitivity threshold of $100 \mathrm{EeV}$ impacting the surface of the Moon with low frequency radio telescopes. In this work we discuss the option to use this effect with LOW Frequency ARay (LOFAR, [3]) for UHECR research, and also to study putative $\mathrm{ZeV}$ neutrinos that could arise from exotic physics [4].

\section{Theory and Principles of Detection}

The low flux of UHECRs around the energy cutoff requires that we have a very large detection area. In the 1960s Askaryan proposed using the Moon as a detector volume [5], though the initial assumption was to put detectors on the Lunar surface. Later, Dagkesamanskii and Zheleznykh [6] suggested the use of Earth-based radio telescopes to search for lunar pulses produced by UHECRs or neutrinos interacting with the Moon. The near-surface of the Moon which is about 19 million $\mathrm{km}^{2}$ implies that even for a flux as low as one particle per $\mathrm{km}^{2}$ per century culminates into an impact every 3 minutes. The rather large average Earth-Moon distance (of $\approx 3.88 \times 10^{5} \mathrm{~km}$ ) puts a very high energy threshold for particle detection.

Askaryan was the first to suggest that coherence builds up when the wavelength (in $\mathrm{MHz}-\mathrm{GHz}$ frequency range) of the radiation emanating from a particle cascade in a dense medium is of the order of the typical physical dimensions of the shower or cascade. The electric field strength scales with the excess charged particle and the power in the radiation grows as a square of the shower energy. This makes the Lunar Askaryan technique suitable for detecting UHECRs or neutrinos. By pointing an Earth-based radio telescope to the Moon, short pulses, of duration of a nanosecond, can be detected. In the case of no detection, an upper limit on cosmic-ray and neutrino flux can be set, constraining models. This method was attempted by many experiments with no detection as yet [79]. All these experiments except the NuMoon experiment (at Westerbork) were carried out at higher frequencies (above a $1 \mathrm{GHz}$ ). At higher frequencies, we are limited to the rim of the Moon as most the signals suffer total internal reflection away from the rim. However, at lower frequencies (i.e. LOFAR frequency), the wavelength of the radiation is comparable to the longitudinal dimensions of the shower making the coherent emission more isotropic with a larger angular spread around the Cherenkov angle (i.e. cherenkov cone scales inversely with frequency [10] eq. 19). This implies that observing at lower frequencies leads to a significant increase in the effective aperture, with the whole Lunar surface contributing to the detector volume [11]. However, these observations have higher energy threshold. An analytic parameterization for effective aperture is used in this work 
(refer to $[10,12]$. Work is in progress to simulate the effective aperture). Also, the radio absorption length increases towards lower frequencies, hence radio waves induced by neutrinos at greater depth are detectable. Radio waves from cosmic rays on the other hand suffers lesser attenuation and hence are easier to detect. At the neutrinos energies considered in this work, the neutrinos are unable to go through the Moon as they have larger cross sections.

\section{Detection with LOFAR}

LOFAR (Low Frequency Array) is currently the largest radio telescope operating at the lowest frequencies. It has 51 stations across 8 different European countries. Each station consist of two types of antennas: high band (HBA, 110-240 MHz) and low band (LBA, 10-90 MHz) antennas (8000 antennas in total). For this work, the HBA antennas at the core of LOFAR were used, the signal is digitized by an 8 bit ADC at $200 \mathrm{MHz}$ sampling rate. Analogue filters are applied directly to the inputs to suppress any signal outside $110-190 \mathrm{MHz}$ frequency window. The digitized signal is then split into 512 subbands via polyphase filter (PPF) followed by 1024 point Fast Fourier transform (FFT). Polyphase filtering is an efficient way of resampling and and applying a multi-channel finite impulse response (FIR) filter while reducing the sampling rate. This reduces the time resolution from a few nanoseconds to a few micro-seconds. The next step after channel separation is the digital phase rotation beam-former, which adds the various channelized signals after multiplication with a complex phase factor representing the geometrical delay required for beam-forming towards a certain user-defined direction.

The effective area $\left(A_{\text {eff }}=\min \left(\frac{1}{3} \lambda^{2}, 1.5625\right)\right.$ per antenna) of six half LOFAR stations (used for observation) is $\approx 3138 \mathrm{~m}^{2}$ (taking into account the Moon's elevation at time of observation and central frequency; $141 \mathrm{MHz}$ ). Hence, the system equivalent flux density (background) for one radio antenna is $360 \mathrm{Jy}$ and its equivalent RMS spectral electric field for this band-width is $0.0029 \mu \mathrm{V} / \mathrm{m} / \mathrm{MHz}$ (see eq. 10 in [13] and system temperature is taken as $430 \mathrm{~K}$ [13]).

For this work, a GPU-based observation pipeline has been developed to process the observation data in real-time. The following steps were taken in analysing the data:

1. Tied-array Beams: An array of antennas can be used a spatial filters canceling the gain in certain directions through destructive interference and enhancing the gain in others through constructive interference. This coherent stacking of the gain of an antenna is certain user defined direction is termed beam forming. By beam-forming, the sensitivity or directivity in certain directions is greatly enhanced. This is done by adding the appropriate time delays or phase delays (consequence of time shifting property of Fourier transform) in the frequency domain to signals from the each element of the phased array, the energy cancels out in undesirable directions and energy from the other directions adds up coherently

Beam formed data from 6 half LOFAR core stations are coherently added together to form 49 smaller tied-array beams covering the entire surface of the Moon. These 49 tied-array beams are formed on $7 \times 7$ grid over the lunar surface. The beam size for LOFAR half station in different frequency beams is computed and displayed in figure 1 . The average beam size over the LOFAR bandwidth is $\approx 4^{\circ}$. 


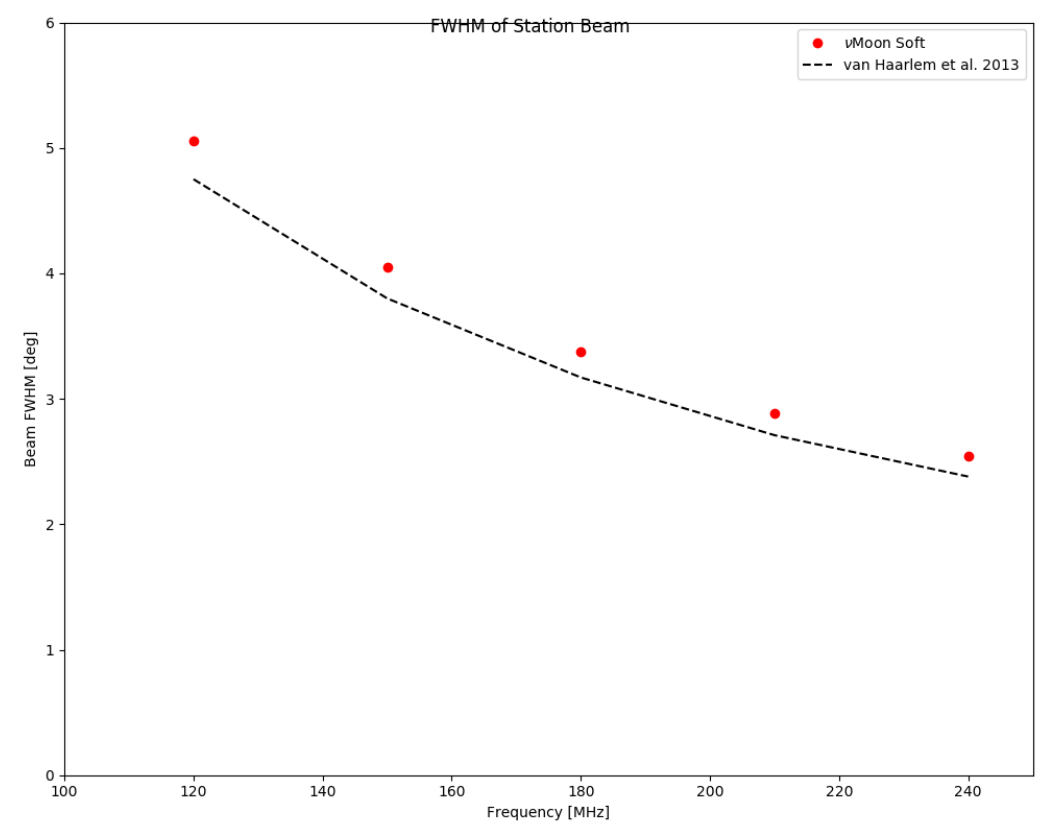

Figure 1: Full width half maximum of a LOFAR half station in degrees as a function frequency in $\mathrm{MHz}$.

Improving sensitivity towards weaker signals requires increasing the number of beam elements and also forming many smaller beams across the entire surface of the Moon. Increasing the beam elements in effect decreases the beam size. Only 49 tied-array beams - as shown in figure 4 - are formed and used in this work because of the limited-bandwidth between LOFAR computers and DRAGNET (this a remote computer cluster on which the analysis software is running [14]).

2. Polyphase Filter (PPF) Inversion: $P P F$ inversion is done to obtain the full nano-second time resolution of the signal since the pulse duration of a typical Lunar pulse is on the order of a few nano-seconds. The efficiency of PPF inversion is about $97 \%$ since the edges of the signal are poorly reconstructed. Hence samples close to the edges have been excluded in this analysis (i.e. only $97 \%$ of observation time will be considered). The details of this process can be found in [15].

3. Ionospheric Dedispersion: The ionosphere is dispersive and hence introduces a frequency dependent delay on the radio waves propagating through it. This stretches the pulse out leading to a temporal broadening of the pulse on timescales given as $\delta_{\mathrm{t}}=1.34 \times 10^{-7} \times$ $S T E C \times\left(v_{l o}^{-2}-v_{h i}^{-2}\right) . \quad v_{l o}$ and $v_{h i}$ are the receiver's minimum and maximum frequency. The STEC is in units of electrons per $\mathrm{m}^{2}$. Consequently, this reduces the pulse's amplitude. Since triggering is done on the pulse amplitude, there is a need to recover the original pulse amplitude by correcting for this effect. Dispersion in time domain can be seen as a phase factor in the frequency domain and depends on the total electron content of the ionosphere;

$$
H(f)=e^{-i \omega \delta_{t}}
$$




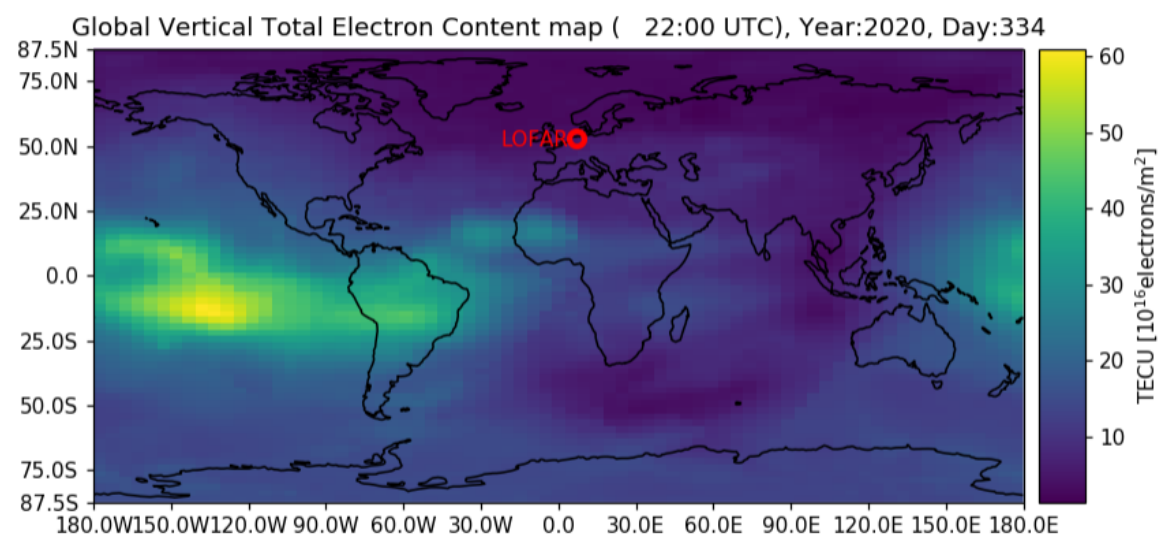

Figure 2: Plot of the gloabal total electron content of the ionosphere in units of TECU (total electron content unit).

The slant total electron content (STEC) is the vertical total electron content (VTEC) of the ionosphere corrected for the Moon's elevation. The VTEC values used in this project was provided by IonSAT research group ${ }^{1}$. The global total electron content (TEC) map for the day (29 November, 2020) and time (22 UTC) of the observation is display in figure 2 and the location of the LOFAR observatory marked in red with a VTEC value of 5.9 TECU $\left(1 \mathrm{TECU}=10^{16}\right.$ electrons $\left./ \mathrm{m}^{2}\right)$. The de-dispersion of a signal is carried out by multiplying the frequency dependent phase factor, $H(f)$ with the signal in the frequency domain (apply FFT), and then converting back into the time domain (inverse FFT).

4. Radio Frequency Interference (RFI) Removal: These are narrow band emissions that can account for a significant fraction of the received power and have to be filtered out. A baseline [16] is fitted to the spectrum of the data and frequency bin values exceeding the baseline by $50 \%$ are set to zero.

5. Pulse Detection and triggering: A trigger is registered when $P_{5}$ value exceeds $5 . P_{5}$ is a unit-less parameter that defines the strength of the pulse. $\mathrm{P}_{x}$ and $\mathrm{P}_{y}$ are the powers in the $x$ and $y$ polarizations respectively.

$$
P_{5}=\frac{\sum_{5 \text { samples }} P_{x}}{\left\langle\sum_{5 \text { samples }} P_{x}\right\rangle}+\frac{\sum_{5 \text { samples }} P_{y}}{\left\langle\sum_{5 \text { samples }} P_{y}\right\rangle}
$$

Due to the uncertainty; 0.1 TECU in the measured STEC value, pulse width is further increased hence the choice of 5 samples over which the power is spread for a nyquist sampled band-limited pulse. To suppress RFIs that escaped the RFI filtering algorithm requires a secondary trigger logic or cuts. These cuts can only be design by first making background measurements. Base on the preliminary measurements (will be discussed in a later section), cuts are currently being developed (results will be shown in a later publication).

\footnotetext{
${ }^{1}$ Technical Univ. of Catalonia (UPC-IonSAT)
} 

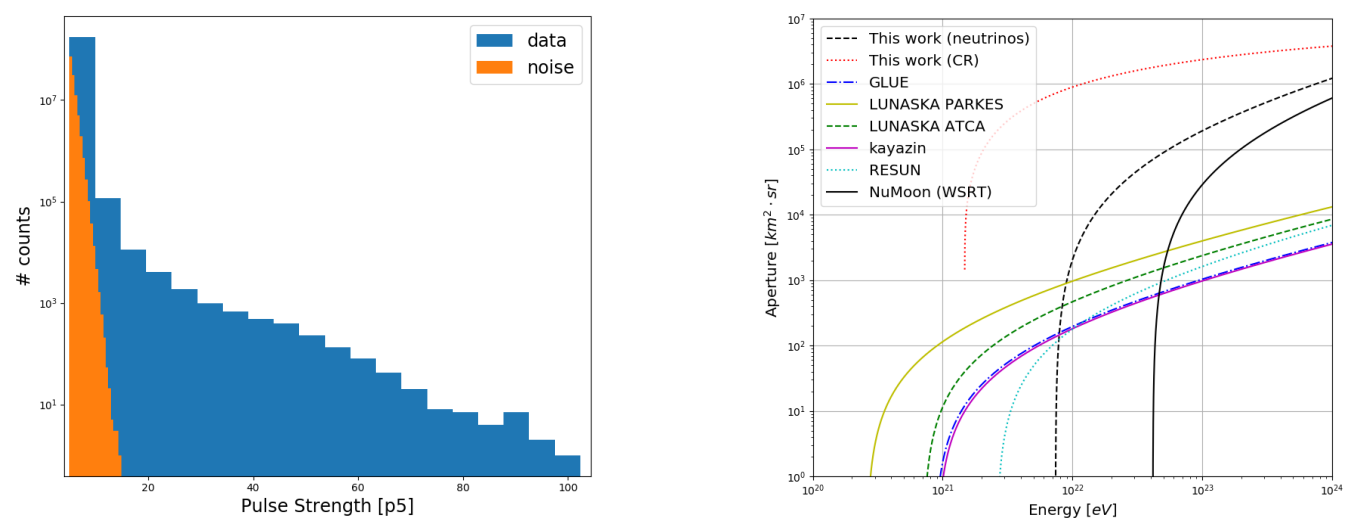

Figure 3: Left: Distribution of $\mathrm{P}_{5}$ values without any veto. Gaussian noise in orange and data in blue. Right: Effective aperture for neutrinos and CR using parameterization in [10, 12].

\section{Observation and Results}

Observations were carried out on the 29 November, 2020 at 22 UTC for one minute using 6 half LOFAR core stations in the Netherlands. All beams were directed at the Moon located at $18.69^{\circ} \mathrm{DEC}$ and $61.28^{\circ} \mathrm{RA}$. The 6 stations used are: 'CS003HBA0', 'CS013HBA0', 'CS030HBA0','CS031HBA0', 'CS301HBA0' and 'CS401HBA0'. The data is scanned for values of $\mathrm{P}_{5}$ that exceed 5 and these are recorded as triggers. Figure 3 (left) displays the distribution of $\mathrm{P}_{5}$ values for both the observed data (in blue) and pure Gaussian noise (in orange) without any selection criteria. The orange points in figure 3 (left) correspond to the amount of triggers expected if the background is pure Gaussian noise. At this point, RFI has already been filtered out and dispersion corrected. The STEC value during observation is 7.3 TECU. The maximum pulse strength detected for 2.3 seconds of the analysed data is $\mathrm{P}_{5}=102.37$. It is however not the goal of this preliminary work to produce a stringent constraint. The goal is to measure the background and to be able to develop a selection criteria that reduces the trigger rate. This step is an essential step before using a real-time trigger as intended.

Some of the strongest background pulses were studied and an example is figure 4 (right). As shown, the observed signal in all beams have nearly similar maximum strength ( $\mathrm{P}_{5}$ value). This suggest RFI or strong signal from the horizon. This feature has been reproduced in simulation for a signal coming from the horizon into the station beams. Figure 4 (left) displays a simulated signal pulse from the Moon center. The distribution maximum pulse strength across all 49 tied-array beams is non-uniform with the maximum occurring at the center of the analysis/tied-array beams. This difference in the distribution of the maximum pulse strength across all beams for an actual Lunar signal and a background can be exploited to make a selection criteria/cuts.

No pulse with strength exceeding $\mathrm{P}_{5}=102.37$ was found and this translates $102.37 \times 360=$ $37 \mathrm{kJy}(0.027 \mu \mathrm{V} / \mathrm{m} / \mathrm{MHz})$ assuming a $100 \%$ detection efficiency. This null detection over a given time, $t$, is converted to a useful upper bound on the neutrino and cosmic ray flux using a modelindependent differential flux upper limit 3 (at 90\% confidence level and assuming Poisson counting statistics) as shown in 5: 

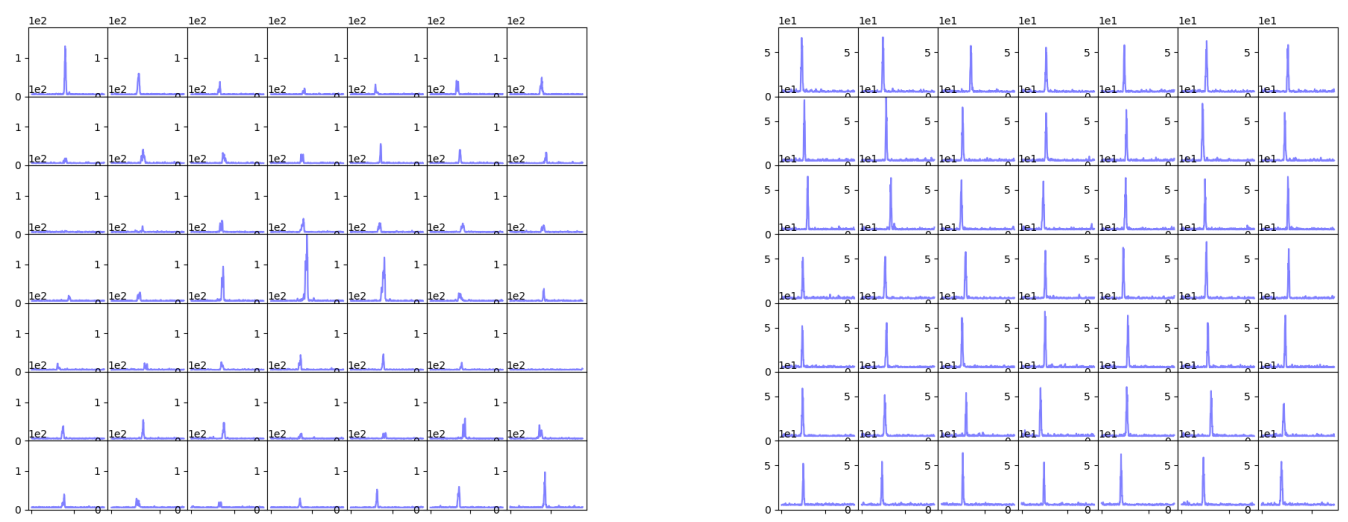

Figure 4: Two different plots of $\mathrm{P}_{5}$ values above 5 of 49 tied-array beams. Left: simulation of a signal pulse from the Moon. Right: background pulse in data.

$$
F(E, t)<2.3 \frac{E}{t A_{e}(E)}[9]
$$

$A_{e}(E)$ is the energy dependent effective aperture, the area times the solid angle through which incident neutrinos or cosmic rays are detectable and is taken from [10,12]. Figure 3 (right) displays the effective aperture for 6 half LOFAR core station. The Moon's elevation at the time of observation is considered. $E$ is primary energy of the UHE particle. In figure 5, the upper limit on cosmic rays and neutrino flux (left and right respectively) have been established for 2.3 seconds of the observed data analysed. For ease of comparison, upper limit calculations of previous experiments were done based on similar parameterizations used in this work and results shown in figure 5 (see legend). The result from this preliminary work demonstrates that the Lunar detection technique works. Results are also shown for an upper limit when the observation time is extrapolated to 50 hours and the maximum $\mathrm{P}_{5}$ value has been lower by a factor of 5 (see **This work (max $\mathrm{p} 5$ reduced by 5 and 50 hrs) in legend of figure 5). By reducing the maximum $\mathrm{P}_{5}$ value via additional selection criteria, the energy threshold is lowered closer to the GZK energies for UHECRs. Increasing the observation time on the other hand lowers the upper limit as shown in figure 5.

\section{Discussion and Outlook}

The next phase of this work will be to develop a triggering layer and then proceed to carrying out longer hours of observation and include more stations in the observation (additional 4 stations). This should provide the best upper limit at the highest energies if no Lunar pulse is detected.

\section{References}

[1] G. T. Zatsepin and V. A. Kuzmin, "Upper limit of the spectrum of cosmic rays," JETP Lett., vol. 4, pp. 78-80, 1966.

[2] A. M. Hillas, "The Origin of Ultra-High-Energy Cosmic Rays," araa, vol. 22, pp. 425-444, Jan. (1984).

[3] M. P. van Haarlem and et al., "Lofar: The low-frequency array," Astronomy Astrophysics, vol. 556, p. A2, Jul 2013. 

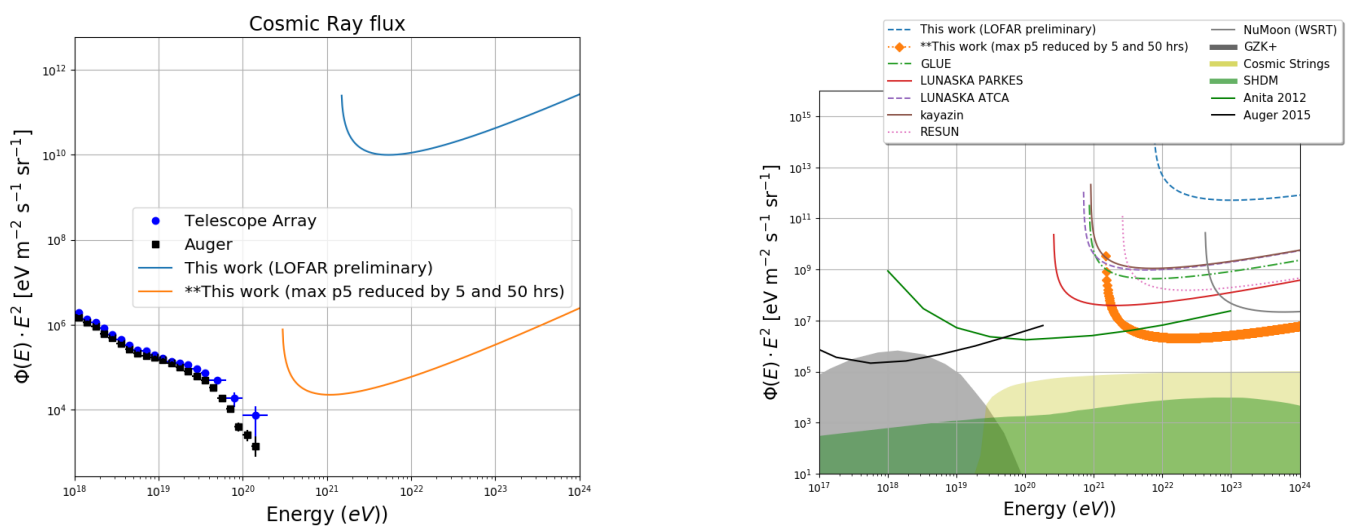

Figure 5: Upper limit on the flux of UHE particles from different experiments. (Left) cosmic ray flux. (Right) neutrino flux.

[4] T. Winchen and et al., "Properties of the Lunar Detection Mode for ZeV-Scale Particles with LOFAR," 2019.

[5] G. Askary'an, "Excess negative charge of an electron-photon shower and the coherent radio emission from it," Zhur. Eksptl'. i Teoret. Fiz, (1961).

[6] R. Dagkesamanskii and I. Zheleznykh, "Radio-astronomy method for detecting neutrinos and other elementary particles of superhigh energy," Sov. Phys. JETP 50, (1989).

[7] P. W. Gorham, C. L. Hebert, K. M. Liewer, C. J. Naudet, D. Saltzberg, and D. Williams, "Experimental limit on the cosmic diffuse ultrahigh energy neutrino flux," Phys. Rev. Lett., vol. 93, p. 041101, Jul 2004.

[8] "Ultra-high-energy cosmic ray and neutrino detection using the moon," Nuclear Physics B - Proceedings Supplements.

[9] T. Jaeger, R. Mutel, and K. Gayley, "Project resun, a radio evla search for uhe neutrinos," Astroparticle Physics, vol. 34, p. 293-303, Dec 2010.

[10] T. J. K.G. Gayley, R.L. Mutel, "Analytic aperture calculaton and scaling laws for radio detection of lunar-target uhe neutrinos," (2009).

[11] O. S. et al., "Optimal radio window for the detection of ultra-high-energy cosmic rays an neutrinos off the moon," (2006).

[12] M. R. Y.S.Jeong, "Radio cherenkov signals from the moon: neutrinos and cosmic rays," (2011).

[13] J. Bray, "The sensitivity of past and near-future lunar radio experiments to ultra-high-energy cosmic rays and neutrinos," Astroparticle Physics, vol. 77, p. 1-20, Apr 2016.

[14] DRAGNET, “https://www.astron.nl/lofarwiki/doku.php?id=dragnet:start."

[15] T. Winchen and et al., "Search for cosmic particles with the moon and lofar," EPJ Web of Conferences, vol. 135, p. 04003, 2017.

[16] Q. Zeng, X. Chen, X. Li, J. L. Han, C. Wang, D. J. Zhou, and T. Wang, "Radio frequency interference mitigation based on the arpls and sumthreshold method," 2020. 